\title{
Carfentanil: a weapon of mass destruction
}

\author{
Steven L. Shafer, MD
}

Received: 12 December 2018/Accepted: 12 December 2018/Published online: 14 January 2019

(C) Canadian Anesthesiologists' Society 2019

Which would you choose as a weapon of mass destruction: a thermonuclear bomb or carfentanil?

Carfentanil ${ }^{1,2}$-it's not even close.

North Korea recently tested a thermonuclear bomb with a mass of approximately $300 \mathrm{~kg}^{3}$ and an estimated yield of 250 kilotons. $^{4}$ If exploded over a major urban center, a thermonuclear bomb of this weight would kill approximately 2 million people ${ }^{5}$-i.e., about 10,000 people per kilogram of bomb mass. Fortunately, thermonuclear bombs are incredibly difficult to make. Delivered by intercontinental ballistic missiles, they invite likely obliteration of any country insane enough to launch a nuclear attack.

Now consider carfentanil. Carfentanil is about twice as potent as sufentanil, ${ }^{6}$ and 20 -fold more potent than fentanyl. ${ }^{7}$ I confirmed potency of carfentanil with Dr. James Frost (James Frost, personal communication, September 14, 2018), who used $\left[{ }^{11} \mathrm{C}\right]$-carfentanil as an opioid ligand in human positron emitted tomography (PET) studies over the past three decades. ${ }^{8-11}$ These PET studies initially used $0.1 \mu \mathrm{g} \cdot \mathrm{kg}^{-1}$, but the investigators reduced the dose because of mild ventilatory depression. They found that $0.02 \mu \mathrm{g} \cdot \mathrm{kg}^{-1}$ produced almost no discernable effect. This is compatible with a 20 -fold potency relative to fentanyl where $2 \mu \mathrm{g} \cdot \mathrm{kg}^{-1}$ of fentanyl would cause mild ventilatory depression, and $0.4 \mu \mathrm{g} \cdot \mathrm{kg}^{-1}$ would have virtually no discernable effect. These updated carfentanil calculations contrast with what is frequently (and thus erroneously) stated as being 100 -fold more potent than

\section{S. L. Shafer, MD ( $\square)$}

Department of Anesthesiology, Perioperative and Pain Medicine, Stanford University, 300 Pasteur Drive, Stanford, CA 94305,

USA

e-mail: steven.shafer@stanford.edu fentanyl. ${ }^{12,13}$ Consistent with the early reports by Janssen ${ }^{6}$ and Mather, ${ }^{7}$ three decades of human PET studies with $\left[{ }^{11} \mathrm{C}\right]$-carfentanil studies suggest this was an exaggeration.

Nevertheless, an opioid 20-fold more potent than fentanyl represents a significant threat. If $1,000 \mu \mathrm{g}$ of fentanyl would be a lethal dose in the absence of ventilatory support (anybody disagree?), an equivalent lethal dose of carfentanil would be just $50 \mu \mathrm{g}$. As documented by Leen and Juurlink in this issue of the Journal, ${ }^{14}$ "the Royal Canadian Mounted Police seized 1 $\mathrm{kg}$ of carfentanil." One kilogram of carfentanil represents 20 million fatal $50 \mu \mathrm{g}$ doses, enough to kill half the population of Canada. On a per kilogram basis, carfentanil is arguably 2000-fold deadlier than a thermonuclear bomb.

The Figure shows the death rates from synthetic opioids (primarily fentanyl) in the United States from 19992017. ${ }^{15}$ I modeled the data as a linear increase from 1999 2010, with a superimposed logarithmic increase from 2010 onwards (black line). Logarithmic growth ends badly, as suggested by the extrapolation (red line) from 2017-2022. The model suggests synthetic opioid overdoses will double approximately every 14 months, reaching over a million deaths/year by 2022 . This probably won't happen, because drug dealers will die of opioid overdose, the population of potential users will shrink, and society will find better ways of preventing addiction. Nevertheless, the trends show the grim potential for synthetic opioid overdoses.

Carfentanil has arrived, appearing throughout the United States, ${ }^{16-19}$ Europe $^{20,21}$ and Canada. ${ }^{22}$ The Figure is based primarily on fentanyl deaths. Synthetic opioid deaths are likely to accelerate with carfentanil, which must be diluted 100 million-fold to create a sellable $10 \mu \mathrm{g}$ dose. How many drug dealers have the pharmaceutical grade equipment to aliquot carfentanil with enough accuracy to not eventually kill their customers? 

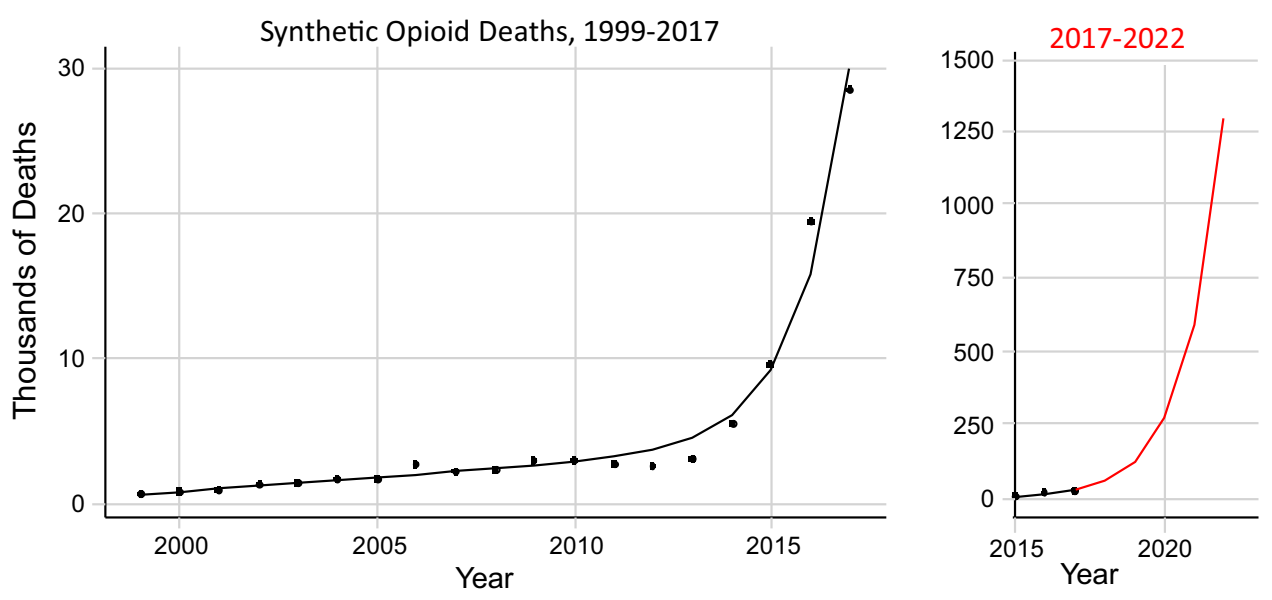

Figure The graph to the left shows synthetic opioid deaths in the United States from 1999-2017, based on data from the Centers for Disease Control. ${ }^{15}$ The black line represents a superimposed additive fit of the data and superimposed exponential fit starting in 2010. The graph to the right shows the model extrapolation (red line) from 2017-2022

Trying to block the supply of carfentanil is a fool's errand. Paul Janssen clearly explained the synthesis of carfentanil, which is no more difficult than synthesizing fentanyl. ${ }^{23}$ Decades ago I lost a close friend to fentanyl he synthesized in his desk drawer. This isn't rocket science. Domestic production will likely replace imported sources. Thousands of doses can be mailed in overnight express envelopes. Policing has failed miserably to stop the supply of heroin and fentanyl. Despite the success of the Royal Canadian Mounted Police busting dealers with staggering amounts of carfentanil, ${ }^{24}$ there is no chance a policefocused approach will successfully interdict carfentanil supply.

To eliminate the threat to users, and to our society, we must eliminate the demand. This isn't news to anybody. What is different today is the urgency. Look back at the Figure. We can't wait a year or two. The opioid of mass destruction is here today.

We must understand the causes of substance use disorder. $^{25,26} \mathrm{We}$ must provide individuals at risk with the social, medical, psychiatric, and spiritual support to address the root causes that place them at risk of opioid use. We need to treat opioid abuse disorder as a disease, which means providing the research and therapeutic investment we make in every other significant threat to public health (i.e., cancer, heart disease, diabetes). We need to think outside the box. Maybe we need to decriminalize drug use, so we can get addicts into treatment. Going further, maybe we should we create centres for supervised injection of pharmaceutical grade opioids, perhaps even offered in exchange for addicts agreeing to inpatient
Le graphique de gauche présente le nombre de décès par opioïde de synthèse aux États-Unis de 1999 à 2017 d'après les données des Centers for Disease Control. ${ }^{15}$ La ligne noire représente la superposition de l'addition des données et de l'ajustement exponentiel à partir de 2010. Le graphique de droite présente l'extrapolation du modèle (ligne rouge) pour les années 2017 à 2022

detoxification, thus treating their disorder while drying up the market for fentanyl and carfentanil?

Every life is an endless conveyor belt of stress, challenge, and pain. We must provide those at risk with tools to cope with life's challenges without resorting to opioids. Everyone needs support. Everyone needs security. Everyone needs something to live for. This will be expensive. This will never be "solved."

To policy makers who would rather buy military hardware, I'm sorry to share bad news, but opioid addiction, turbocharged with carfentanil, represents an existential threat. We must defeat it by combining sustained resources with steadfast dedication and political resolve.

The risk from nuclear weapons is no different. Nukes pose an ongoing, existential threat to our society. Recognizing this, we have committed vast sustained resources and political resolve to reduce the risk of nuclear annihilation. Carfentanil is arguably more dangerous than nuclear weapons. It has arrived; the death toll is rising; and pound for pound, it is orders of magnitude more dangerous than a thermonuclear bomb.

The time to act is now.

\section{Carfentanil: une arme de destruction massive}

Que choisiriez-vous comme arme de destruction massive : une bombe thermonucléaire ou le carfentanil?

Le carfentanil ${ }^{1,2}$ sans l'ombre d'un doute. 
La Corée du Nord a récemment testé une bombe thermonucléaire ayant une masse d'environ $300 \mathrm{~kg}^{3}$ avec une puissance explosive estimée de 250 kilotonnes. ${ }^{4} \mathrm{Si}$ elle explosait au-dessus d'une grande métropole, une bombe thermonucléaire de ce poids tuerait approximativement 2 millions de personnes, ${ }^{5}$ soit près de 10000 personnes par kilo de bombe. Heureusement, les bombes thermonucléaires sont incroyablement difficiles à fabriquer. Emportées par des missiles balistiques intercontinentaux, elles entraîneraient le probable anéantissement de tout pays suffisamment dément pour déclencher une attaque nucléaire.

Voyons maintenant le carfentanil. Le carfentanil est environ deux fois plus puissant que le sufentanil, ${ }^{6}$ et 20 fois plus puissant que le fentanyl. ${ }^{7} \mathrm{~J}^{\prime}$ ai confirmé la puissance du carfentanil avec le $\mathrm{D}^{\mathrm{r}}$ James Frost (communication personnelle, 14 septembre 2018), qui a utilisé le $\left[{ }^{11} \mathrm{C}\right]$-carfentanil comme ligand opioïde dans les études par tomodensitométrie à émission de positons (TEP) chez l'homme au cours des trente dernières années. ${ }^{8-11}$ Ces études de TEP ont utilisé initialement des doses de 0,1 $\mu \mathrm{g} \cdot \mathrm{kg}^{-1}$, mais les investigateurs les ont diminuées en raison de dépression respiratoire légère. Ils ont établi qu'une dose de $0,02 \mu \mathrm{g} \cdot \mathrm{kg}^{-1}$ n'entraînait quasiment aucun effet identifiable. Cela est compatible avec une puissance 20 fois supérieure à celle du fentanyl dont $2 \mu \mathrm{g} \cdot \mathrm{kg}^{-1}$ provoquerait une dépression respiratoire légère et 0,4 $\mu \mathrm{g} \cdot \mathrm{kg}^{-1} \mathrm{n}$ 'aurait virtuellement aucun effet identifiable. Ces calculs actualisés concernant le carfentanil contrastent avec ce qui est fréquemment (et donc faussement) cité : le carfentanil n'est pas 100 fois plus puissant que le fentanyl. ${ }^{12,13}$ Trois décennies d'études avec le $\left[{ }^{11} \mathrm{C}\right]$ carfentanil utilisé pour des TEP chez l'homme vont dans le même sens que les premières publications de Janssen ${ }^{6}$ et Mather, ${ }^{7}$ suggérant qu'il s'agissait d'une exagération.

Il n'en reste pas moins qu'un opioïde 20 fois plus puissant que le fentanyl représente une menace significative. Si $1000 \mu \mathrm{g}$ devait être une dose létale de fentanyl en l'absence de soutien ventilatoire (qui n'est pas d'accord?), la dose létale équivalente de carfentanil serait juste $50 \mu \mathrm{g}$. Comme Leen et Juurlink l'indiquent dans ce numéro du Journal, ${ }^{14}$ " la Gendarmerie royale du Canada a saisi $1 \mathrm{~kg}$ de carfentanil ». Un kilogramme de carfentanil représente 20 millions de doses mortelles de $50 \mu \mathrm{g}$, suffisamment pour tuer la moitié de la population du Canada. Par kilo, le carfentanil est indiscutablement 2000 fois plus dangereux qu'une bombe thermonucléaire.

La Figure montre les taux de décès par opioïdes de synthèse (principalement le fentanyl) aux États-Unis de 1999 à 2017. ${ }^{15}$ J'ai modélisé les données sous forme d'augmentation linéaire à partir de 1999-2010, avec une superposition de l'augmentation logarithmique à partir de 2010 (ligne noire). La croissance logarithmique se termine mal, à en croire l'extrapolation pour la période 2017 à 2022 (ligne rouge). Le modèle suggère que le nombre de victimes de surdoses d'opioïdes de synthèse va doubler approximativement tous les 14 mois pour atteindre plus d'un million de morts par an d'ici 2022. Cela n'arrivera probablement pas parce que les revendeurs de drogue mourront de surdose d'opioïdes, la population d'utilisateurs potentiels va diminuer et la société trouvera de meilleurs moyens de lutter contre la dépendance. Néanmoins, les tendances montrent le risque sinistre de surdoses par opioïdes de synthèse.

Le carfentanil est arrivé, apparaissant aux ÉtatsUnis, ${ }^{16-19}$ en Europe ${ }^{20,21}$ et au Canada. ${ }^{22}$ Le graphique de la Figure s'appuie principalement sur les décès dus au fentanyl. La fréquence des décès dus aux opioïdes de synthèse va sans doute s'accélérer avec le carfentanil qui doit être dilué 100 millions de fois pour créer une dose vendable de $10 \mu \mathrm{g}$. Combien de revendeurs de drogues disposent de l'équipement de qualité pharmaceutique pour faire des doses aliquotes de carfentanil avec une précision suffisante pour ne pas tuer leurs clients?

Tenter de bloquer la production de carfentanil est illusoire. Paul Janssen a clairement expliqué comment synthétiser le carfentanil : ce n'est pas plus difficile que de synthétiser du fentanyl. ${ }^{23}$ Il y a quelques décennies, j'ai perdu un ami proche à cause du fentanyl qu'il synthétisait dans le tiroir de son bureau. Ce n'est pas sorcier. La production locale remplacera probablement les importations. Des milliers de doses peuvent être expédiées dans des enveloppes et délivrées le lendemain matin par les sociétés de messagerie. Les tentatives des autorités à bloquer la fourniture d'héroïne et de fentanyl ont misérablement échoué. En dépit du succès de la Gendarmerie royale du Canada qui a coincé des revendeurs avec une quantité effarante de carfentanil, ${ }^{24}$ il n'y a quasiment aucune chance de voir un succès dans l'arrêt du trafic de carfentanil à partir des actions de la police.

Pour éliminer la menace qui pèse sur les utilisateurs et sur notre société, nous devons éliminer la demande. Il n'y a là rien de neuf pour personne. Ce qui est différent aujourd'hui, c'est l'urgence à laquelle nous sommes confrontés. Regardez une nouvelle fois la figure. Nous ne pouvons pas encore attendre un an ou deux. L'opioïde de destruction massive est ici, aujourd'hui parmi nous.

Nous devons comprendre les troubles d'addiction de substance. $^{25,26}$ Nous devons apporter aux personnes à risque le soutien social, médical, psychiatrique et spirituel dont ils ont besoin pour aborder les causes profondes qui les exposent au risque d'utilisation des opiö̈des. Nous devons traiter le trouble d'abus des opioïdes comme une maladie, ce qui signifie de fournir les moyens financiers pour la recherche et le traitement comme nous le faisons pour toutes les autres menaces significatives sur la santé du 
public (par exemple, le cancer, les maladies cardiaques, le diabète). Nous devons avoir une réflexion plus globale. Peut-être, devrions-nous décriminaliser la consommation de drogues pour permettre aux personnes dépendantes de recevoir un traitement. Allant encore plus loin, peut-être devrions-nous créer des centres d'injections supervisées d'opioïdes de qualité pharmaceutique, éventuellement offerts en échange d'une cure de désintoxication par les toxicomanes ce qui permettrait de traiter leur trouble tout en éliminant le marché du fentanyl et du carfentanil?

Chaque vie est une suite sans fin de stress, de défis et de douleur. Nous devons fournir à ceux qui sont à risque les outils pour faire face aux défis de la vie sans avoir à recourir aux opioïdes. Tout le monde a besoin de soutien. Tout le monde a besoin de sécurité. Tout le monde a besoin d'avoir un but dans l'existence. Cela coûtera cher. Cela ne sera jamais « résolu».

Aux décideurs politiques qui achèteraient plutôt du matériel militaire, j'ai le regret de leur annoncer la mauvaise nouvelle : la toxicomanie aux opioïdes, suralimentée par la carfentanil, représente une menace existentielle. Nous devons la vaincre en combinant des ressources constantes avec une détermination sans faille et une volonté politique.

Le risque créé par la menace nucléaire n'est pas différent. Ces bombes représentent une menace constante, existentielle pour notre société. Le reconnaissant, nous avons consacré continuellement d'importantes ressources et une volonté politique à la réduction du risque d'anéantissement nucléaire. Le carfentanil est incontestablement plus dangereux qu'une arme nucléaire. Il est arrivé; le nombre de morts augmente; et poids pour poids, sa dangerosité est d'une magnitude considérablement supérieure à celle d'une bombe thermonucléaire.

Il faut agir. Maintenant.

Support This work was supported by departmental resources

Conflict of interest The author has no conflicts of interest to report for the topic of this editorial.

Editorial responsibility This submission was handled by Dr. Hilary P. Grocott, Editor-in-Chief, Canadian Journal of Anesthesia.

Soutien Ce travail a été soutenu par les ressources du département.

Conflit d'intérêts L'auteur n'a aucun conflit d'intérêts à déclarer pour le thème de cet éditorial.

Responsabilité éditoriale Cet article a été traité par le Dr Hilary P. Grocott, Rédacteur en chef, Journal canadien d'anesthésie.

\section{References}

1. The Mackenzie Institute Security Matters. Carfentanil Street Drug and Weapon of Mass Destruction Available on Canadian Streets. Available from URL: http://mackenzieinstitute.com/carfentanilstreet-drug-weapon-mass-destruction-available-canadian-streets/ (accessed December 2018)

2. Di Gregorio D. The Use of Carfentanil as WMD-the issue hiding within the current opioid crisis. CBRNe Portal. Available from URL: https://www.cbrneportal.com/the-use-of-carfentanil-as-awmd-the-issue-hiding-within-the-current-opioid-crisis/ (accessed December 2018)

3. Jane's 360. North Korea bargains with nuclear diplomacy. Available from URL: https://www.janes.com/images/assets/111/ 75111/North_Korea_bargains_with_nuclear_diplomacy.pdf (accessed December 2018).

4. NORSAR. The nuclear explosion in North Korea on 3 September 2017: a revised magnitude assessment. Available from URL: https://www.norsar.no/press/latest-press-release/archive/the-nucl ear-explosion-in-north-korea-on-3-september-2017-a-revised-ma gnitude-assessment-article1548-984.html (accessed December 2018).

5. Bergstrom $S$. On the demographic impact of nuclear war. Population and Development Review 1983; 9: 562-8.

6. Janssen PA. Potent, new analgesics, tailor-made for different purposes. Acta Anaesthesiol Scand 1982; 26: 262-8.

7. Mather LE. Clinical pharmacokinetics of fentanyl and its newer derivatives. Clin Pharmacokinet 1983; 8: 422-46.

8. Frost JJ, Wagner HN Jr, Dannals RF, et al. Imaging opiate receptors in the human brain by positron tomography. J Comput Assist Tomogr 1985; 9: 231-6.

9. Frost JJ, Douglass KH, Mayberg HS, et al. Multicompartmental analysis of $[11 \mathrm{C}]$-carfentanil binding to opiate receptors in humans measured by positron emission tomography. J Cereb Blood Flow Metab 1989; 9: 398-409.

10. Frost JJ, Mayberg HS, Sadzot B, et al. Comparison of [11C]diprenorphine and [11C]carfentanil binding to opiate receptors in humans by positron emission tomography. $\mathrm{J}$ Cereb Blood Flow Metab 1990; 10: 484-92.

11. Frost JJ. PET imaging of the opioid receptor: the early years. Nucl Med Biol 2001; 28: 509-13.

12. PubChem. Carfentanil. Available from URL: https://pubchem. ncbi.nlm.nih.gov/compound/carfentanil (accessed December 2018).

13. Wikipedia. Carfentanil. Available from URL: https://en. wikipedia.org/wiki/Carfentanil (accessed December 2018).

14. Leen JL, Juurlink DN. Carfentanil: a narrative review of its pharmacology and public health concerns. Can J Anesth 2019; 66. DOI: https://doi.org/10.1007/s12630-019-01294-y.

15. Centers for Disease Control and Prevention. Data Brief 329. Drug Overdose Deaths in the United States, 1999-2017. Available from URL: https://www.cdc.gov/nchs/data/databriefs/ db329_tables-508.pdf\#page $=4$ (accessed December 2018).

16. Shanks $K G$, Behonick GS. Detection of carfentanil by LC-MSMS and reports of associated fatalities in the USA. J Anal Toxicol 2017; 41: 466-72.

17. Daniulaityte $R$, Juhascik MP, Strayer KE, et al. Overdose deaths related to fentanyl and its analogs - Ohio, January-February 2017. MMWR Morb Mortal Wkly Rep 2017; 66: 904-8.

18. O'Donnell JK, Halpin J, Mattson CL, Goldberger BA, Gladden $R M$. Deaths involving fentanyl, fentanyl analogs, and U-47700 10 states, July-December 2016. MMWR Morb Mortal Wkly Rep 2017; 66: 1197-202.

19. O'Donnell J, Gladden RM, Mattson CL, Kariisa M. Notes from the field: overdose deaths with carfentanil and other fentanyl 
analogs detected - 10 states, July 2016-June 2017. MMWR Morb Mortal Wkly Rep 2018; 67: 767-8.

20. Hikin L, Smith PR, Ringland E, Hudson S, Morley SR. Multiple fatalities in the North of England associated with synthetic fentanyl analogue exposure: detection and quantitation a case series from early 2017. Forensic Sci Int 2018; 282: 179-83.

21. Elliott SP, Hernandez Lopez E. A series of deaths involving carfentanil in the UK and associated post-mortem blood concentrations. J Anal Toxicol 2018; 42: e41-5.

22. Government of Canada. Overview of national data on opioidrelated harms and deaths. Available from URL: https://www. canada.ca/en/health-canada/services/substance-use/problematicprescription-drug-use/opioids/data-surveillance-research/harmsdeaths.html (accessed December 2018).

23. Janssens F, Torremans J, Janssen PA. Synthetic 1,4disubstituted-1,4-dihydro-5H-tetrazol-5-one fentanyl: alfentanil (R 39209), a potent, extremely short-acting narcotic analgesic. J Med Chem 1986; 29: 2290-7.

24. Toronto Sun; Doucet C. Carfentanil: the drug of mass destruction. Available from URL: https://torontosun.com/news/local-news/ carfentanil-the-drug-of-mass-destruction (accessed December 2018).

25. Samuelson ST, Bryson EO. The impaired anesthesiologist: what you should know about substance abuse. Can J Anesth 2017; 64: 219-35.

26. Lefebvre LG, Kaufmann IM. The identification and management of substance use disorders in anesthesiologists. Can J Anesth 2017; 64: 211-8.

Publisher's Note Springer Nature remains neutral with regard to jurisdictional claims in published maps and institutional affiliations. 